\title{
Rotura traumática de riñón en herradura
}

\author{
Pascual Samaniego M, Bravo Fernández I, Ruiz Serrano M, Ramos Martín JA, \\ Lázaro Méndez J, García González A.
}

Servicio de Urología. Hospital San Pedro de Alcántara. Cáceres.

Actas Urol Esp 2006; 30 (4): 424-428

\section{RESUMEN}

\section{ROTURA TRAUMÁTICA DE RIÑÓN EN HERRADURA}

Entre un tercio y la mitad de los riñones en herradura son asintomáticos y se diagnostican incidentalmente. Esta anomalía congénita de fusión se ha mostrado como condición predisponente a la lesión renal ante un traumatismo abdominal, aunque con frecuencia el grado de afectación renal no precise cirugía. Más excepcional es el hallazgo de fractura de riñón en herradura, requiriéndose un estudio diagnóstico completo para un manejo adecuado a sus características cuando la cirugía está indicada.

Se aporta el caso de un varón de 15 años con riñón en herradura no previamente conocido, que presentó una fractura de localización atípica polar superior y mesorrenal derecha, tras un traumatismo abdominal de baja intensidad. El correcto diagnóstico prequirúrgico permitió una cirugia diferida con preservación del polo renal inferior derecho y del istmo del riñón en herradura.

Las características del trauma, la desproporción de la repercusión clínica y la búsqueda de posibles anomalías congénitas asociadas y de signos radiológicos compatibles, pueden orientar esta rara posibilidad diagnóstica, confirmándose el TAC con contraste como la mejor prueba de imagen.

Palabras clave: Traumatismo, riñón en herradura, cirugía conservadora.

\section{ABSTRACT}

\section{TRAUMATIC FRACTURE OF A HORSESHOE KIDNEY}

One-third to one-half of all patients with horseshoe kidney are asymptomatic and the condition is found incidentally. This congenital renal anomaly has shown as a predisponent condition for renal injury in blunt abdominal trauma, but often the degree of injury has a nonoperative therapy. Horseshoe kidney rupture is an exceptional pathology that require a complete diagnostic study to make an adequate management when surgical therapy is indicated.

We present a fifteen year-old-male with previously unsuspected horseshoe kidney that suffered an atipic right upper-pole and mesorrenal kidney fracture after low velocity impact blunt abdominal trauma. A correct presurgical diagnose let a deferred surgical approach with rigth lower pole and horseshoe renal isthmus preservation. The trauma conditions, an excesive clinic manifestation, a clinical investigation about known congenital simultaneous anomallies and typical radiological signs, can suggest this infrequent patology. Computed tomography provide the best radiological information.

Key words: trauma, horseshoe kidney, conservative surgery.

$\mathrm{E}^{\mathrm{t}}$ riñón en herradura es la anomalía congénita de fusión más frecuente con una incidencia de $1 / 400$ a 1/800 recién nacidos vivos, afectando 2 veces más a varones ${ }^{1}$. Sus características le confieren la predisposición a su lesión ante un traumatismo abdominal incluso de baja intensidad ${ }^{2}$.

El diagnóstico incidental de un riñón en herradura como consecuencia de su rotura tras un traumatismo abdominal cerrado es excepcio$\mathrm{nal}^{3}$, puede ser dificultoso y resulta básico para un adecuado tratamiento quirúrgico lo más conservador posible cuando éste es necesario, dadas sus particulares características anatómicas, de localización y la variabilidad de su vascularización ${ }^{1,4}$. La lesión más frecuente ocurre a nivel del istmo renal ${ }^{5}$. 
A continuación revisamos los signos clínicos y radiológicos que es necesario investigar por su asociación al riesgo de presentar una lesión sobre esta anomalía renal congénita no previamente conocida, en el contexto de un traumatismo abdominal cerrado, aportando un nuevo caso de rotura de riñón en herradura de localización atípica polar superior y mesorrenal derecha.

\section{CASO CLÍNICo}

Varón de 15 años de edad, sin antecedentes medicoquirúrgicos de interés, que ingresa de urgencia tras recibir un golpe fortuito de moderada intensidad en flanco derecho practicando balonmano. Refiere dolor continuo en hemiabdomen derecho no irradiado, un vómito alimenticio, e inicio de hematuria macroscópica en la hora posterior.

A la exploración se encuentra consciente, afebril, con moderada palidez cutáneo-mucosa, dolor, matidez y palpación de masa en hipocondrio y vacío derechos con defensa abdominal voluntaria y ruidos hidroaéreos conservados. Evaluación neurológica normal. Presión arterial: 80/40 mmHg. Frecuencia cardiaca: 80 lat/min.

La analitica de sangre inicial presentaba Hb:12,2 g/dl, Ht: 37,3\%, 22.200 leucocitos $/ \mathrm{mm}^{3}$, neutrófilos $82 \%$ y creatinina: $0,6 \mathrm{mg} / \mathrm{dl}$, siendo el estudio de coagulación normal. El análisis de orina mostraba hematuria macroscópica, proteínas >300 mg/dl, glucosa $250 \mathrm{mg} / \mathrm{dl}$, nitritos positivos y un nivel alto de bilirrubina.

En la radiografía simple de abdomen se apreciaba un borramiento de la línea renopsoas derecha. La ecografía abdominal informaba de rotura renal derecha con hematoma retroperitoneal hasta la fosa iliaca derecha, contusión del segmento posterior del lóbulo hepático derecho con mínima cantidad de líquido libre perihepático y cavidad vesical con múltiples coágulos, siendo el riñón izquierdo y el bazo normales.

Con el paciente monitorizado y estable hemodinamicamente tras reposición hidroelectrolítica, se realizó un TAC abdomino-pélvico con contraste, identificándose unos riñones en herradura con fractura del polo superior del riñón derecho y afectación calicilar con extravasado de contraste perirrenal, posible fístula arteriovenosa y gran hematoma retroperitoneal superando la linea media hacia el espacio perirrenal izquierdo con desplazamiento de estructuras retro e intraperitoneales, (Figs. 1 y 2).

Ante este nuevo dato diagnóstico, la estabilidad clínica y analítica seriada y la cesión de la hematuria con preservación de constantes y diuresis, el paciente se traslada a UCI, permitiéndonos una planificación adecuada para una cirugía conservadora. Se transfunden 4 concentrados de hematies de forma progresiva, no bajando el hematocrito de $28 \%$.

A pesar de la evolución favorable del hematoma retroperitoneal, ante la reaparición intermitente de hematuria de escasa cuantía y la posibilidad de una fístula vascular, se programa la intervención a los 16 días del ingreso. Se realizó una lumbotomía derecha con evacuación del hematoma organizado perirrenal y extracción de

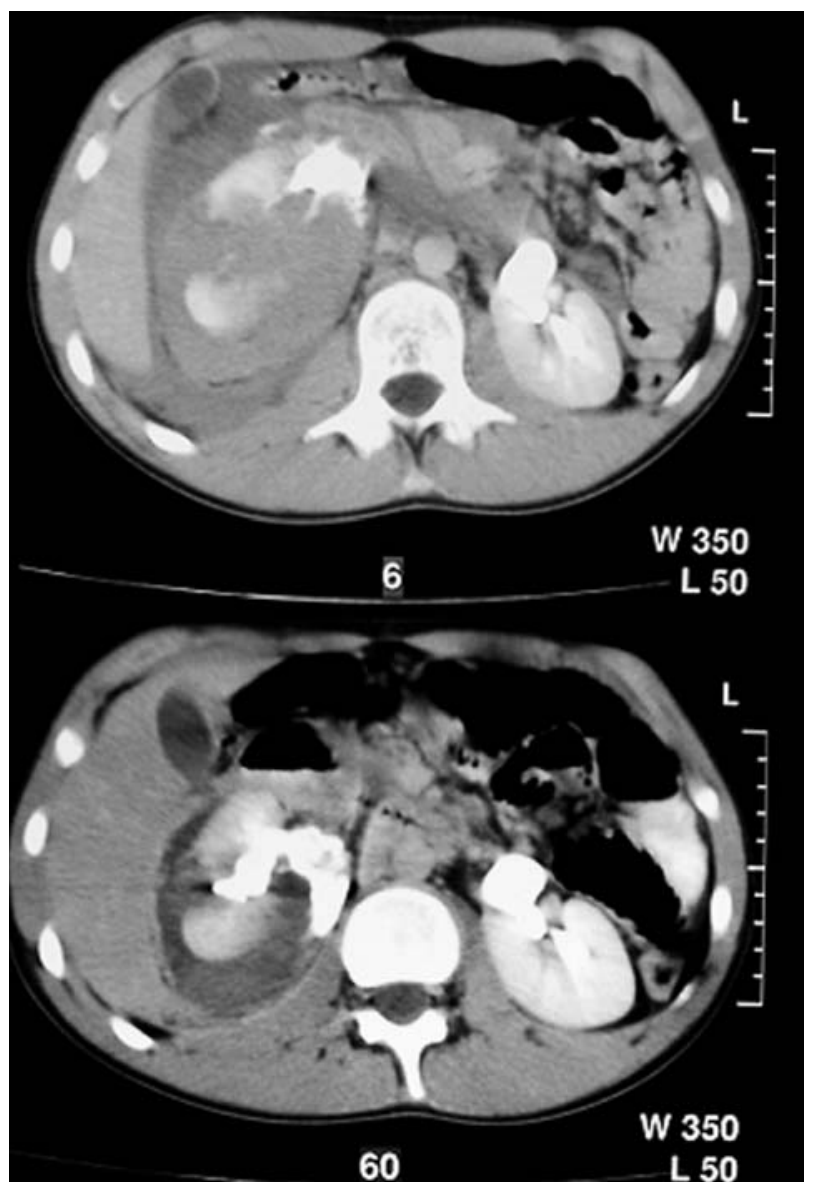

FIGURA 1. TAC abdominal con contraste intravenoso. Fractura del polo superior y mesorrenal derecho de riñón en herradura afectando a la vía urinaria con extravasación de contraste, grado IV de la AAST. Posible asociación de fistula arteriovenosa. 


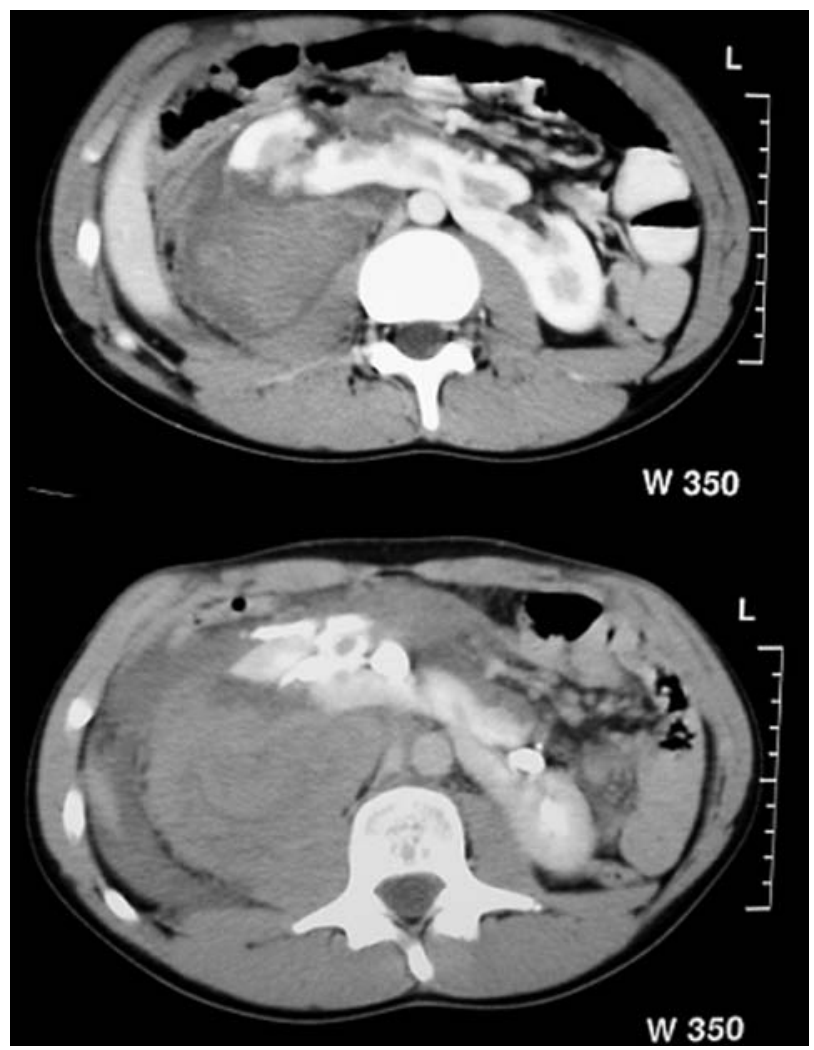

FIGURA 2. TAC abdominal con contraste intravenoso. Fractura de riñón en herradura con preservación del istmo renal parenquimatoso. Gran hematoma retroperitoneal derecho.

tres fragmentos de parénquima renal correspondientes al polo superior y a la zona mesorrenal externa derecha desvitalizados. Se colocó un catéter ureteral derecho por pielotomía y un catéter percutáneo Malecot $14 \mathrm{Ch}$., con sutura del grupo calicial inferior y de la zona mesorrenal.

Doce días después el paciente presentó febrícula y débito de orina por el drenaje, por lo que se colocó un catéter doble $\mathrm{J}$ ureteral derecho, resultando ineficaz. Manteniendo el drenaje, la sonda vesical y un control ambulatorio ecográfico de ausencia de colecciones, se resolvió conservadoramente la fístula urinaria calicial postquirúrgica al cabo de dos semanas. Tres años después, el paciente presenta una función renal analítica y urográfica normal, (Fig 3).

$\mathrm{El}$ informe anatomopatológico de la nefrectomía parcial derecha por rotura traumática de riñón en herradura, reveló ausencia de patología glomérulo-tubular, con extensa hemorragia y necrosis intersticial y presencia de glándula suprarrenal derecha fragmentada.

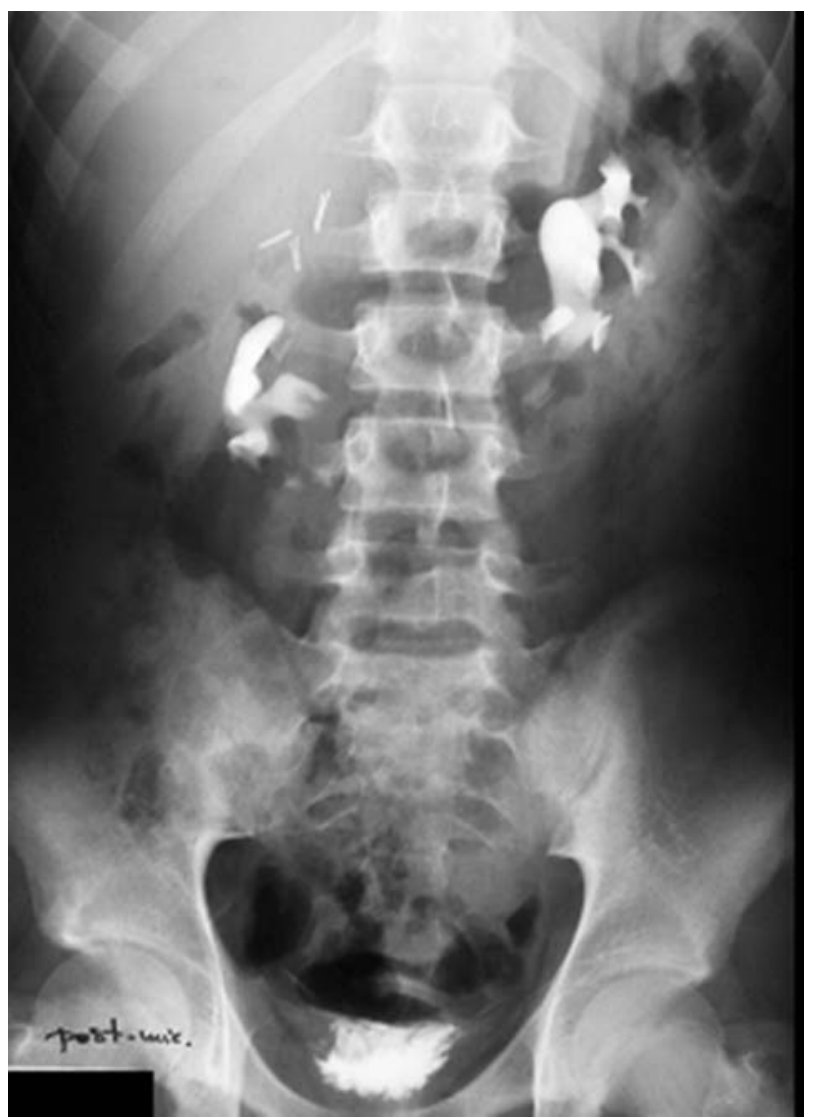

FIGURA 3. Urografia intravenosa tras cirugia conservadora. Polo inferior derecho del riñón en herradura con funcionalidad conservada.

\section{DISCUSIŌN}

La incidencia del riñón en herradura en la población general es del $0.25 \%^{2,3}$. No se conocen claros determinantes genéticos ${ }^{1}$ para esta anomalía congénita de fusión para la cual se han propuesto dos teorías etiopatogénicas en función del tipo de tejido que constituya el istmo. Cuando éste es de tejido fibroso, se habla de la teoría mecánica de fusión que se produciría durante la organogénesis entre la $4^{\mathrm{a}}$ y la $6^{\mathrm{a}}$ semana de gestación con el embrión entre 5 y $12 \mathrm{~mm}$, por fusión de los polos inferiores de las masas metanéfricas o blastemas renales después de la implantación de las yemas ureterales ${ }^{2}$. Cuando el istmo es parenquimatoso (85\%), se ha postulado que la fusión sería el resultado de un proceso teratogénico de migración anormal de las células nefrogénicas posteriores que se unirian para formar el istmo. Ese evento teratogénico podría explicar también el aumento de incidencia de ciertas neoplasias como el tumor de Wilms o el 
tumor carcinoide, con un riesgo relativo 2 y 62 veces superior respectivamente, ambos afectando en la mitad de los casos al istmo renal. Igualmente podría ser responsable de la frecuente asociación de anomalías congénitas, de las cuales las genitourinarias aparecen hasta en las 2/3 partes de los pacientes. Entre ellas, la estenosis de la unión pieloureteral (35\%), el reflujo vesicoureteral (50\%), la duplicidad ureteral (10\%), la criptorquidia y el hipospadias en un $4 \%$ de los varones y el septo vaginal y el útero bicorne en el 7\% de las mujeres. También se conocen anomalías óseas, gastrointestinales, mielomeningocele y cardiovasculares como la comunicación interventricular. Algunos síndromes genéticos como la trisomía 18 (20\%) o el síndrome de Turner (60\%) pueden asociar riñón en herradura ${ }^{6}$.

La fusión, que afecta a los polos inferiores en más del 90\% de los casos, impide la migración de los riñones por encima del origen de la arteria mesentérica inferior, quedando ectópicos a cualquier nivel entre la pelvis y dicha arteria, con el istmo habitualmente por delante de los grandes vasos y entre la $3^{\text {a }}$ y la $5^{\underline{a}}$ vértebras lumbares ${ }^{1}$. La fusión también impide la normal rotación renal quedando los polos inferiores mediales con la pelvis renal en situación anterior y una inserción ureteral alta.

Esta anomalía congénita por si sola no afecta a la supervivencia, aunque como se ha mencionado, asocia una mayor propensión a desarrollar patologias tumorales, infecciosas y traumáticas en virtud de múltiples factores como su localización y anatomía, su embriogénesis, la presencia del istmo y la variabilidad de su vascularización. Ante un traumatismo abdominal cerrado, asociado al $90 \%$ de las lesiones renales ${ }^{7}$, como consecuencia de esta situación inferior del riñón en herradura que pierde la protección de las últimas costillas y de su disposición anterior a las vértebras lumbares, existe un riesgo aumentado para la lesión renal principalmente por compresión o fractura del istmo contra la columna lumbar ${ }^{1,2,8}$. Así, el mecanismo traumático relacionado con más frecuencia, es anteroposterior ${ }^{1}$.

Nuestro paciente presentó una lesión renal grado IV según la escala de severidad de lesión renal de la American Association for the Surgery of Trauma ${ }^{9}$ (AAST). Su localización inhabitual a nivel polar superior y mesorrenal derecha sin afectar al istmo, se relaciona con el mecanismo traumático sobre el flanco derecho y explica la lateralización del dolor abdominal, mientras que en la lesión del istmo el dolor suele referirse a la zona periumbilical. Se cumplían las características que se han asociado al paciente tipo con riesgo de presentar un riñón patológico desconocido, como son el traumatismo único, con baja asociación de lesión de otros órganos, el impacto de baja velocidad ${ }^{10}$ y la hematuria macroscópica desproporcionada respecto a la baja intensidad del trauma ${ }^{8}$. Esto sugiere que la susceptibilidad a una lesión renal mayor ante un traumatismo leve, no se limita al istmo del riñón en herradura. Debemos valorar clínicamente estos signos de sospecha, más aún en la población pediátrica, así como las posibles anomalías congénitas asociadas que nos inclinen a buscar radiológicamente el diagnóstico del riñón en herradura, ya que modificará el planteamiento de la actitud terapéutica.

La ecografia puede no ser definitiva y la urografia intravenosa, que en situación normal manifiesta signos de sospecha de riñón en herradura como la inversión del eje renal, el descenso de posición, la situación anterior y medial de las pelvis renales, la captación de contraste por el istmo si es parenquimatoso o el cruce del uréter anterior al istmo en la proyección oblicua, se modifican con el traumatismo, el hematoma perirrenal o la ausencia de captación de contraste ${ }^{2,5}$. Por ello el TAC con contraste se confirma como la prueba radiológica de elección ${ }^{11}$ en el paciente hemodinámicamente estable, cuyas indicaciones bien establecidas son: hematuria macroscópica, hematuria microscópica asociada a tensión arterial sistólica $<90 \mathrm{mmHg}$ o a lavado peritoneal diagnóstico positivo ${ }^{12}$. Es el mejor método para clasificar la severidad de la lesión renal, la presencia de extravasado urinario, de lesión vascular y de hemorragia perirrenal, permitiendo el diagnóstico de las anomalías estructurales insospechadas como el riñón en herradura y la disposición de su vascularización de cara a un posible abordaje quirúrgico. Sólo el 30\% presentan una arteria renal única bilateral. La mayoría presentan combinaciones de arteria renal única o múl- 
tiple con aporte supletorio de la aorta, las arterias iliacas o la arteria mesentérica inferior ${ }^{1,13}$. El istmo recibe aporte vascular único de la aorta en el $65 \%$ de los casos. En el resto su irrigación puede proceder de la arteria iliaca común o de la mesentérica inferior.

Los urólogos hemos adoptado una actitud cada vez más conservadora en el manejo del traumatismo renal cerrado en las lesiones de grado IV, ya que la pérdida de sangre y de parénquima renal y la necesidad de cirugía abierta son menores con el tratamiento conservador o con cirugía diferida respecto a la cirugía abierta inmediata ${ }^{14-16}$. La presencia de extravasado urinario por si solo en la lesión renal de grado IV no es indicación de cirugía, siempre que la pérdida de parénquima no supere el $50 \%$, ya que puede resolverse espontáneamente hasta en el $87 \%$ de los casos ${ }^{17}$, como ocurrió con la fistula calicial postquirúrgica de nuestro paciente a pesar de la respuesta inicial desfavorable tras colocar un catéter doble J.

$\mathrm{El}$ fracaso del tratamiento conservador inicial por sangrado diferido como en el caso que presentamos, se produce en menos del 10\% de los pacientes, existiendo estudios que asocian factores de riesgo independientes de mayor probabilidad de fallo del manejo no quirúrgico en el trauma renal, como el requerimiento de transfusiones sanguíneas o la estimación de más de $300 \mathrm{ml}$ de líquido libre en el $\mathrm{TAC}^{18}$.

La posibilidad de una actitud conservadora inicial ante la sospecha clínica de una lesión traumática en riñón anómalo, nos permitió un adecuado estudio radiológico de la infrecuente localización de la fractura de un riñón en herradura. Ante el requerimiento de cirugía diferida, se optó por el abordaje de lumbotomía dada la lateralidad de la lesión frente a la incisión media abdominal habitualmente recomendada en los casos más frecuentes con afectación del istmo, consiguiendo un resultado favorable de cirugia conservadora.

\section{REFERENCIAS}

1. Allen RC. Horseshoe Kidney. (Artículo electrónico. Ultima revisión: 14/10/04). Disponible en http://www.emedicine. com/med/topic2860.htm.

2. Murphy JT, Borman KR, Davidson I. Renal autotransplantation after horseshoe kidney injury: a case report and literature review. J Trauma 1996; 40(5):840-844.
3. Daudia A, Hassan TB, Ramsay D. Trauma to a horseshoe Kidney. J Accid Emerg Med 1999;16(6):455-456.

4. Ward VL, Khorasani R. Horseshoe Kidney. BrighamRAD Professional Education. (Artículo electrónico. Ultima revisión: 03/09/96). Disponible en: http://brighamrad. harvard.edu/Cases/bwh/hcache/196/full.html.

5. Muruamendiaraz V, Martín Benito JL, Castellanos González L, Castaño González-Coto D, Alonso Sainz F. Traumatismo de riñón en herradura. Actas Urol Esp 1979; 3(6):345-348.

6. Soto M, Bachiller J, Rodríguez-Rubio RI, Rodríguez-Rubio F. Contribución al estudio del riñón en herradura. Formación continuada en Urología. Volumen 5, №2 Pulso Ediciones S.A.,1999.

7. Robert M, Drianno N, Muir G, Delbos O, Guieter J. Management of major blunt renal lacerations: surgical or nonoperative approach?. Eur Urol 1996; 30(3): 335-339.

8. Chopra P, St-Vil D, Yazbeck S. Blunt renal trauma-blessing in disguise?. J Pediatr Surg 2002;37(5):779-782.

9. Moore EE, Shackford SR, Pachter HL, McAninch JW, Browner BD, Champion HR, et al. Organ injury scaling spleen, liver and kidney. J Trauma 1989;29(12):1664-1666.

10. Schmidlin FR, Iselin CE, Naimi A, Rohner S, Borst F, Farshad M, et al: The higher injury risk of abnormal kidneys in blunt renal trauma. Scand J Urol Nephrol 1998; 32(6):388-392.

11. Kuo RL, Eachempati SR, Makhuli MJ, Reed II RL. Factors affecting management and outcome in blunt renal injury. World J Surg 2002;26(4):416-419.

12. Harris AC, Zvirewich CV, Lyburn ID, Torreggiani WC, Marchinkow LO. CT findings in blunt renal trauma. Helping the trauma surgeon. RSNA Scientific Assembly. Radiographics 2001;21 Spec No:S201-14.

13. Boullier J, Chehval M, Purcell MH. Removal of a multicystic half of a horseshoe kidney: significance of preoperative evaluation in identifying abnormal surgical anatomy. $\mathrm{J}$ Pediatr Surg 1992;27(9):1244-1246.

14. Knudson MM, Maull KI. Nonoperative management of solid organ injuries. Past, present, and future. Surg Clin North Am 1999;79(6):1357-1371.

15. Danuser H, Wille S, Zöscher G, Studer UE. How to treat blunt kidney ruptures: primary open surgery or conservative treatment with deferred surgery when necessary?. Eur Urol 2001;39(1):9-14.

16. Santucci RA, McAninch JW, Safir M, Mario LA, Service S, Segal MR. Validation of the American Association for the Surgery of Trauma organ injury severity scale for the kidney. J Trauma 2001;50(2):195-200.

17. Brandes SB, McAninch JW. Reconstructive surgery for trauma of the upper urinary tract. Urol Clin North Am 1999;26(1):183-199.

18. Velmahos GC, Toutouzas KG, Radin R, Chan L, Demetriades D. Nonoperative treatment of blunt injury to solid abdominal organs. Arch Surg 2003;138(8):844-851.

Dr. M. Pascual Samaniego

E-mail: mpaski10@yahoo.es

(Trabajo recibido el 19 de octubre 2005) 